\title{
On various forms of bipolarity in flexible querying
}

\author{
Didier Dubois ${ }^{1}$ Henri Prade ${ }^{1}$ \\ ${ }^{1}$ IRIT, CNRS \& Université Paul Sabatier, 31062 Toulouse Cedex 09
}

\begin{abstract}
The paper discusses the modeling of "if possible" in requirements of the form " $A$ and if possible $B$ ". We distinguish between two types of understanding: either i) $A$ and $B$ are requirements of the same nature and are viewed as constraints with different levels of priority, or ii) they are of different nature (only $A$ induces constraint(s) and $B$ is only used for breaking ties among items that are equally satisfying $A$ ). We indicate that the two views are related to different types of bipolarity, and discuss them in relation with possibilistic logic. The disjunctive dual of the first view (" $A$ or at least $B$ ") is then presented in this logical setting. We also briefly mention the idea of an extension of the second view where $B$ may refer both to bonus conditions or malus conditions that may increase or decrease respectively the interest in an item satisfying $A$.
\end{abstract}

Keywords: preference query; bipolarity; possibilistic logic; fuzzy set.

\section{Introduction}

Preference and flexible queries have attracted a considerable interest in different circles and at different epochs among databases researchers $[37,32,35,6$, $7,21,15,31,14,34,40,41,36]$. There may exist slightly different motivations for using flexible queries. One may want to introduce some tolerance by tacitly enlarging crisp queries to similar requested items. One may more often try to express preferences. The expectation is then to both i) rank-order retrieved items according to the extent to which they are satisfactory, and ii) try to avoid empty answers by not restricting the query to the profile of the most preferred items (which may not exist in the database). The interested reader may consult [30] for a comparative overview of different approaches to preference queries in database systems, ranging from early mainstream databases proposals that distinguish between mandatory conditions and secondary conditions, or use similarity relations, to fuzzy set-based approaches involving gradual membership functions and priorities, to Pareto ordering-based preference models (where no commensurability hypothesis between the satisfaction degrees pertaining to the different attributes involved is needed), and finally to conditional ceteris paribus preferences (where the request may take the form of comparative preferences stated in specific contexts). More generally, a broad panorama of approaches to the representation and the handling of preferences in operations research, databases, and artificial intelligence can be found in [17].

In the following, we focus the discussion on issues related to the idea of bipolarity in the expression of preference queries, an idea that has been recently developed $[10,8,33,1]$. The idea of bipolarity refers to the distinction between what is regarded as positive and what is regarded as negative, or in other words, between what is found satisfactory and what is rejected. Bipolarity may be encountered in preference (or in knowledge) representation under different forms [25]. One may use bipolar univariate scales ranging from what is completely bad to what is completely good. Another form of bipolarity takes place when items are judged according to two independent evaluations on unipolar scales, a positive one for grading what is in favor of the items, and a negative one for what is in disfavor of them. The evaluations may play a symmetric role or not. In the asymmetric case [26], the evaluations are not based on specifications of the same nature.

More specifically, we examine the problem of modeling requests looking for items that satisfy " $A$ and if possible $B$ ". Such requirements have been considered early by database researchers in order to introduce some hierarchy between requested conditions [32]. In such a basic query, $A$ stands for a (possibly compound) condition that should be imperatively satisfied. Thus, (at least) the items for which $A$ is satisfied are "acceptable", but if they also satisfy $B$, they are considered as being "better" answers. Thus, the requirement " $A$ and if possible $B^{\prime \prime}$ has a bipolar flavor, since on the one hand the items not satisfying $A$ are rejected, while on the other hand those satisfying $A$ and $B$ are positively favored among the items satisfying $A$.

This paper is organized as follows. The next section recalls what the conjunctive condition " $A$ and if possible $B^{\prime \prime}$ may mean precisely, when $A$ and $B$ are of the same nature, i.e. play the role of constraints; we also study the disjunctive condition " $A$ or at least $B$ ". In Section 3 we discuss another view of " $A$ and if possible $B$ " where $B$ is no longer of the same nature as $A$, and is only expressing a wish that is used for breaking ties among items that equally satisfy $A$. This latter view appears to be more refined. Moreover, we briefly suggest that wishes may 
be graded on a bipolar univariate scale (rather than on a positive scale), thus allowing either a positive or a negative impact on the ranking of items satisfying a set of constraints equally. In Section 4 we relate the previous concerns to artificial intelligence works and more particularly to possibilistic logic. This paper is a shortened version of a paper to appear [28].

\section{Modeling "and if possible" and "or at least" in terms of weighted constraints}

In the following, conditions " $A$ and if possible $B$ " and " $A$ or at least $B$ " are considered as expressing a hierarchy of constraints. Conditions $A$ and $B$ are assumed to be consistent. We first examine the case where $A$ and $B$ are crisp, then we show how a set of nested conditions " $A_{n}$ and possibly $A_{n-1}$ and $\ldots$ and possibly $A_{1}$ ", where the $A_{i}$ 's are crisp, is naturally associated with a fuzzy set. Lastly, the approach is extended to when $A$ and $B$ are fuzzy. Moreover, another view where "if possible" is understood as "if consistent" is also addressed.

\section{1. "If possible" : crisp case}

Consider for the moment that $A$ and $B$ are crisp conditions that can be modeled by classical subsets. Thus $A$ and $B$ are sets of interpretations that correspond to configurations of attribute values describing potential items.

With a condition of the form " $A$ and if possible $B$ " where $A$ and $B$ are classical subsets we introduce a hierarchy between three sets of (potential) items, namely

- i) those that satisfy $A \cap B$, i.e. the preferred ones,

- ii) those in $A \cap \bar{B}$, which are still acceptable, and

- iii) those in $\bar{A}$, which are fully rejected.

The condition " $A$ and if possible $B$ " could be stated otherwise as " $B$ provided that $A$ ". It is clear that in this approach, the condition " $A$ and if possible $B$ " is equivalent to the condition " $A$ and if possible $A \cap B^{\prime \prime}$.

We could then assume without loss of generality that $A \supseteq B$, since what is better should be normally inside what is acceptable. In case $A$ and $B$ are general unconstrained requests and if $B \cap \bar{A} \neq \emptyset$ (where $\bar{A}$ denotes the complement of $A$ ), it is thus possible to revise $B$ into $B^{\text {revised }}=A \cap B$ in order to have $A \supseteq B^{\text {revised }}$ since we are only interested in items satisfying $A$. In case $B^{\text {revised }}=\emptyset$, no further discrimination can be made among the items satisfying $A$ since no item satisfying $A$ is fully satisfactory. If $B^{\text {revised }}=A$, no discrimination takes place since all items satisfying $A$ are fully satisfactory.

Then we may think of interpreting the condition " $A$ and if possible $B$ " as a pair of nested sets ( $A, B)$, with $A, B \neq \emptyset$, representing the support and the core of a fuzzy set $F$ respectively, where for an item $x$ with description $\partial(x)$ in terms of attribute values, we have the following membership grades: $F(\partial(x))=1$ if $\partial(x) \in A \cap B ; 1>F(\partial(x))>0$ if $\partial(x) \in A \cap \bar{B}$; and $F(\partial(x))=0$ if $\partial(x) \notin A$. Thus, $F(\partial(x))>0$ iff $\partial(x) \in A$. For simplicity we write $F(x)$ instead of $F(\partial(x))$ in the following.

When $x \in A \cap \bar{B}$ we can prescribe some value $\lambda \in[0,1]$ such that $F(x)=\lambda$. Then it can be checked that $F$ is defined by

$$
F(x)=\min (A(x), \max (\lambda, B(x))
$$

where $\lambda$ is a discounting factor ( $A$ has weight 1 , and $B$ has weight $\lambda$ ) and $A(\cdot), B(\cdot)$ are characteristic functions of $A$ and $B$. When $\lambda=0$ (no discounting), we are back to a classical conjunction while if $\lambda=1$ (total discounting of $B$ ) we get $A$, i.e., $B$ is forgotten. This expression comes from the prioritized fusion of possibility distributions, and is a weighted conjunction first suggested by Dubois and Prade [19]. Interpreting $1-\lambda$ as the degree of priority of $B$, it means $A$ must be satisfied with full priority (1) and $B$ with priority $1-\lambda$, so $x \in A$ is satisfactory to degree $\lambda$ if $B$ is violated. This view has been exploited for the possibilistic approach to prioritized constraints [18]. The above equation can also be read as a formal translation of " $A$ and if possible $B^{\prime \prime}$ (where $\max (\lambda, B(y))$ can be seen as an implication connective $a \rightarrow b=\max (1-a, b)$, viewing $\lambda$ as the complement to 1 of the extent to which it is possible to consider $B$ ).

\section{2. "Or at least": Crisp case}

Dual to the conjunctive "and if possible" conditions are disjunctive "or at least" conditions. Several authors $[9,33]$ write "or else" in place of "or at least", but we prefer this latter phrase which is better suggesting the idea of a hierarchy. It corresponds to the ordered disjunction in qualitative choice logic [13]. With a condition of the form " $A$ or at least $B$ " where $A$ and $B$ are classical subsets we introduce another hierarchy between three sets of (potential) items, namely

- i) those that satisfy $A$, which are the preferred ones,

- ii) those in $B \cap \bar{A}$, which are still acceptable, and

- iii) those in $\overline{A \cup B}$, which are fully rejected.

The condition " $A$ or at least $B$ " could be stated otherwise as " $B$ or better $A$ ". It is clear that in this approach, the condition " $A$ or at least $B$ " is equivalent to the condition " $A$ or at least $A \cup B$ ". Again, it is then possible to change $B$ into $A \cup B$ without harming the condition, which means we could assume $A \subseteq B$ without loss of generality. Again we need to define a membership grade $\mu$ if $\partial(x) \in B \cap \bar{A}$. We obtain a fuzzy set $G$ defined by:

$$
G(x)=\max (A(x), \min (\mu, B(x))
$$


so that $\mu$ is the degree of preference of $B$ rather than its priority. It is clear that this is the so-called weighted disjunction [19], that can be read as a formal translation of " $B$, or at least $A$ ". Indeed, one may either insist that "(at least) $A$ and if possible $B$ " is required, or one may state that one would prefer $B$, or if not possible one would accept at least $A$ by default. This expression entertains a close relationship with the weighted minimum.

Some properties are worth noticing. First, it is clear that " $A$ and if possible $B$ " is equivalent to " $A \cap$ $B$ or at least $A$ ", and " $A$ or at least $B$ " is equivalent to " $A \cup B$ and if possible $A$ " (if $\mu=\lambda$ ). This is clear, from eqs. (1) and (2), and mutual distributivity between union and intersection.

So, if $A \supseteq B$ it holds that " $A$ or at least $B$ " is equivalent to " $B$ and if possible $A$ " since then:

$$
F(x)=\max (B(x), \min (\lambda, A(x))=\min (A(x), \max (\lambda, B(x))
$$

Moreover some De Morgan-type laws are valid as follows: "not $(A$ or at least $B)$ " means "not $A$ and if possible not $B$ " (if $\mu=1-\lambda$, using the negation $1-(\cdot)$ for negating fuzzy set $G$ in $(2))$. Indeed, $1-\max (a, \min (1-\lambda, b))=\min (1-a, \max (\lambda, 1-b))$.

\subsection{Fuzzy case}

We may more generally consider pairs $(A, B)$ such that $A$ and $B$ are normalized fuzzy sets, such as $\exists x, y, A(x)=B(y)=1$. The above results and definitions carry over to this context using idempotent connectives such as the fuzzy weighted minimum and maximum [19]. Namely the fuzzy and if possible and or at least conditions yield fuzzy sets $F$ and $G$ defined by equations (1) and (2) respectively, with all membership grades in the unit interval. The fact that we can restrict to nested fuzzy sets $A \supseteq B$, i.e., $\forall y A(y) \geq B(y)$ is due to the following equalities: $\forall \alpha, \beta, \lambda, \mu \in \mathbb{R}$,

$$
\begin{aligned}
\min (\alpha, \max (\beta, \lambda)) & =\min (\alpha, \max (\min (\alpha, \beta), \lambda)) \\
& =\max (\min (\alpha, \beta), \min (\alpha, \lambda)) ; \\
\max (\alpha, \min (\beta, \mu)) & =\max (\alpha, \min (\max (\alpha, \beta), \mu)) \\
& =\min (\max (\alpha, \beta), \max (\alpha, \mu)) ;
\end{aligned}
$$

that ensure the validity of fuzzy extensions of equations (1), and (2) and the equivalence between the following statements in the fuzzy case :

- " $A$ and if possible $B$ " $\Longleftrightarrow " A$ and if possible $A \cap B " \Longleftrightarrow$ " $A \cap B$ or at least $A$ ";

- " $A$ or at least $B$ " $\Longleftrightarrow$ " $A$ or at least $A \cup B$ " $\Longleftrightarrow$ " $A \cup B$ and if possible $A$ ".

In particular, if $A \supseteq B$, i.e., $\forall y A(y) \geq B(y)$, then we retrieve fuzzy extensions of equation (3), which is the median $\operatorname{med}(A(x), \lambda, B(x))$. So, " $A$ and if possible $B$ " can be turned into " $B$ or at least $A$ " (under the condition $A \supseteq B$ of fuzzy inclusion). Note that if $B=A$, we get $F=A$ (whatever the value of $\lambda$ ), as expected since " $A$ and if possible $A$ " means " $A$ " indeed, even if $A$ is fuzzy.

Both "and if possible" conjunctions and "or at least" disjunctions are studied in detail by Bosc and Pivert [9] in a more general axiomatic setting.

\section{4. "If possible" as "if consistent"}

In the case of fuzzy bipolar queries " $A$ and if possible $B$ ", the importance coefficient $\lambda$ may reflect the consistency between $A$ and $B$, interpreting the constraint as " $A$ and, if $B$ is consistent with $A$, $B$ ". Then, one may choose $\lambda=\operatorname{cons}(A, B)=$ $\sup _{y} \min (A(y), B(y))$, which estimates the consistency of $A$ and $B$. This is what has been proposed in $[20,38]$ for the purpose of information fusion. It applies when one of the pieces of information is certain, while the other is a piece of default information. Expressions formally similar to equations (1) and (2) then express the prioritized conjunction and disjunction of $A$ with $B$ (where $A$ has priority over $B$, so we denote it smaller in the expression of the combination):

$(A \cap B)(y)=\min (A(y), \max (1-\operatorname{cons}(A, B), B(y))$

$(A \cup B)(y)=\max (A(y), \min (\operatorname{cons}(A, B), B(y))$.

As can be seen, when cons $(A, B)=1$, regular conjunctions and disjunctions are retrieved. When, $\operatorname{cons}(A, B)=0, A$ is obtained in both cases. In flexible querying, the possible conflict between $A$ and $B$ does not come only from the specifications as in information fusion, but also from the fact that maybe there does not exist items in the database satisfying $B$ (and then $A$ ). Note that $\operatorname{cons}(A, B)$ accounts only for the extensions of $A$ and $B$ on an attribute domain. Taking the supremum on the items $x$ 's such as $y=\partial(x)$ may make $\operatorname{cons}(A, B)$ smaller since there may exist values $y$ of the attribute domain that are not reached by any item $x$ in the database. The second equation is a prioritized disjunction that becomes all the more restrictive as $B$ becomes incompatible with $A$, in which case only $A$ remains. It means that if $B$ is in slight conflict with $A$, then the objects for which $B$ is true are less preferred, although not fully rejected. De Morgan laws hold between these prioritized conjunctions and disjunction. However, this approach becomes trivial if there is an inclusion relation between normalized $A$ and $B$.

\subsection{Nested "if possible" conditions}

Let a fuzzy set $F$ be defined on a finite scale $\alpha_{1}=$ $1>\alpha_{2}>\cdots>\alpha_{n}>\alpha_{n+1}=0$, and consider its level cuts $F_{\alpha_{i}}=\left\{y \mid F(y) \geq \alpha_{i}\right\}$. Clearly, $F_{\alpha_{1}}$ is the core and $F_{\alpha_{n}}$ the support of $F$, and $F_{\alpha_{n}} \supseteq$ $F_{\alpha_{n-1}} \cdots \supseteq F_{\alpha_{1}}$. The membership function of $F$ is 
then obtained from its level cuts as

$$
F(y)=\max _{i=1, n} \min \left(\alpha_{i}, F_{\alpha_{i}}(y)\right)=\min _{i=1, n} \max \left(\alpha_{i+1}, F_{\alpha_{i}}(y)\right)
$$

where $F_{\alpha_{i}}(y)=1$ if $y \in F_{\alpha_{i}}$ and $F_{\alpha_{i}}(y)=0$ otherwise. The first equality is just Zadeh's representation of a fuzzy set in terms of its cuts [39]. It can be viewed as requesting " $F_{\alpha_{1}}$ or at least $F_{\alpha_{2}}$ or $\ldots$ or at least $F_{\alpha_{n}}{ }$. The other one is the decomposition of a fuzzy constraint into crisp prioritized ones [18]. Then it is clear that such a fuzzy set can also be seen as a representation of " $F_{\alpha_{n}}$ and if possible $F_{\alpha_{n-1}}$ and $\ldots$ and if possible $F_{\alpha_{1}}$ ". Indeed an item $x$ will be all the better as its description $\partial(x)$ has a greater degree of membership in $F$, i.e. according to equation (6), belongs to a level cut with a higher value of $\alpha_{i}$, which means that more "if possible" conditions are satisfied by $x$.

When $n=2$, we get:

$$
\begin{aligned}
F(y) & =\max \left(F_{\alpha_{1}}(y), \min \left(\alpha_{2}, F_{\alpha_{2}}(y)\right)\right. \\
& =\min \left(F_{\alpha_{2}}(y), \max \left(\alpha_{2}, F_{\alpha_{1}}(y)\right),\right.
\end{aligned}
$$

which returns 1 if $y \in F_{\alpha_{2}} \cap F_{\alpha_{1}}=F_{\alpha_{1}}, 1>\alpha_{2}>0$ if $y \in F_{\alpha_{2}} \cap \overline{F_{\alpha_{1}}}$, and 0 if $\overline{F_{\alpha_{2}}}$, in agreement with the above representations of " $A$ and if possible $B$ " and " $B$ or at least $A$ "with $A=F_{\alpha_{2}}$ and $B=F_{\alpha_{1}}$.

\section{Modeling "and if possible" in terms of constraints and wishes}

In $[22,24]$, we have proposed and advocated another view of " $A$ and if possible $B$ " where $A$ is still a constraint, while $B$ is only used for breaking ties between items having the same evaluation w.r.t. $A$.

\subsection{Asymmetric handling of bipolar queries}

In this approach, the condition " $A$ and if possible $B$ " means that $B$ can be used only if the condition $A$ leaves indifferent options. Considering the pair of valuations $(A(x), B(x)) \in[0,1]^{2}$ qualifying the merit of object $x$, the preference on the set of objects is defined by means of the lexicographic ranking of these pairs. Namely

$$
\begin{aligned}
(\alpha, \beta) \succ(\gamma, \delta) \Longleftrightarrow & \text { either } \alpha>\gamma \\
& \text { or }(\alpha=\gamma>0 \text { and } \beta>\delta) .
\end{aligned}
$$

Note that the requirement $(A, B)$ is not the same as $(A, A \cap B)$ since if $B\left(x_{1}\right)>A\left(x_{1}\right)>0, B\left(x_{2}\right)>$ $A\left(x_{2}\right)>0$, and $A\left(x_{1}\right)=A\left(x_{2}\right), B\left(x_{1}\right)>B\left(x_{2}\right)$, it is clear that $x_{1}$ is preferred to $x_{2}$ under request $(A, B)$ while they are indifferent with $(A, A \cap B)$. However, objects $x$ such that $A(x)=0$ are rejected whatever the value of $B(x)$. Moreover, it is not clear how to model " $B$ or at least $A$ " in the lexicographic approach, unless we directly define it as " $A \cup B$ and if possible $B^{\prime \prime}$, and compute a lexicographic ranking of the pairs $(\max (A(x), B(x)), B(x))$. But again, it is no longer equivalent to " $B$ or at least $A$ " using a lexicographic ranking of the pairs $(B(x), A(x))$. The latter option is proposed by Liétard et al. [33] (see also their chapter in this book), under the condition $A \supseteq B$. But while under this condition, " $A$ and if possible $B$ " is equivalent to " $B$ or at least $A$ " in the weighted constraint approach, as intuitively expected, this is no longer true in the lexicographic approach, since even if $A \supseteq B$, the lexicographic ranking of the pairs $(B(x), A(x))$ is not the same as lexicographic ranking of the pairs $(A(x), B(x))$.

Yet another idea for further study could be to replace $\cap$ and $\cup$ by leximin and leximax in the processing of $(A, A \cap B)$ and $(A \cup B, B)$.

It is important to notice that this approach where wishes are used for breaking ties between the items that satisfy the constraints only makes sense when the possible levels of satisfaction of the constraints belong to a discrete chain (as it is the case when dealing with nested requirements such as " $A_{n}$ and if possible $A_{n-1}$ and $\ldots$ and if possible $A_{1}$ " and the $A_{i}$ 's are crisp). In case of a continuum of objects valued on $[0,1]$, preferring, e.g., an item $x$ such that $A(x)=0.85$ and $B(x)=0$ to an item $x^{\prime}$ such that $A(x)=0.84$ and $B(x)=1$ would sound highly debatable. Still, in practice, it would be possible to accommodate a request such as "a reasonably priced apartment, if possible close to the train station" with this approach, by discretizing the scale $[0,1]$ into a finite set of levels corresponding to significantly different prices, which requires a granulation step.

\subsection{Comparing the two approaches}

In the elementary case, where $A$ and $B$ are both crisp conditions, the views of $B$ as a weighted constraint, as discussed in the previous section, or as a criterion for breaking ties lead to the same ranking of the considered items, namely first those that satisfy both $A$ and $B$, then those that satisfy $A$ without $B$, and finally those that do not satisfy $A$.

When $A$ and $B$ become fuzzy, the two views are no longer equivalent. This point can be checked by taking $A(y)=\max \left(A_{1}(y), \min \left(\alpha, A_{2}(y)\right)\right.$ and $B(y)=\max \left(B_{1}(y), \min \left(\alpha^{\prime}, B_{2}(y)\right)\right.$, the $A_{i}$ 's and $B_{j}$ 's being crisp subsets. The condition $A \supseteq B$ means here $A_{1} \supseteq B_{1} A_{2} \supseteq B_{2}$ and $\alpha \geq \alpha^{\prime}$ assuming $B_{2} \bigcap A_{2} \neq \emptyset$. Then, by applying equation (3), the view of $A$ and $B$ as constraints yields:

$$
\begin{array}{r}
F(y)=\max \left(B(y), \min \left(\lambda, \max \left(A_{1}(y), \min \left(\alpha, A_{2}(y)\right)\right)\right.\right. \\
=\max \left(B_{1}(y), \min \left(\alpha^{\prime}, B_{2}(y), \min \left(\lambda, A_{1}(y)\right),\right.\right. \\
\left.\min \left(\alpha, \lambda, A_{2}(y)\right)\right)
\end{array}
$$

Note that $A \supseteq B$ means here $A_{2} \supseteq A_{1} \supseteq B_{1}$, $A_{2} \supseteq B_{2} \supseteq B_{1}$ and when $\alpha^{\prime}<\alpha<\lambda$, we get the following evaluations $F(\partial(x))$ for items $x$ :

- 1 if $\partial(x) \in A_{1} \bigcap B_{1}$

- $\lambda$ if $\partial(x) \in A_{1} \bigcap \overline{B_{1}}$

- $\alpha$ if $\partial(x) \in A_{2} \bigcap \overline{A_{1}} \bigcap B_{2}$

- 0 if $\partial(x) \in \overline{A_{2}}$

The other view, where $B$ is used to break ties among items that satisfy $A$ to some extent, leads to rank-order the items in the following way: 
- 1st) those that satisfy $A_{1}$ (and thus $A_{2}$ ) and $B_{1}$

- 2nd) those that satisfy $A_{1}$ (and $A_{2}$ ) and not $B_{1}$ but $B_{2}$

- 3rd) those that satisfy $A_{1}$ (and $A_{2}$ ) and not $B_{2}$ (and thus not $B_{1}$ ),

- 4th) those that satisfy $A_{2}$ (but not $A_{1}$ ) and $B_{2}$,

- 5th) those that satisfy $A_{2}$ but neither $A_{1}$ nor $B_{2}$

- 6th) those that do not satisfy $A_{2}$.

It is clear that this second view is more refined, since we now have 6 distinct layers of items in the above example, instead of 4 with the hierarchically organized constraints approach. Another more sophisticated example illustrating the difference between the two views can be found in $[24,26]$.

\subsection{Positive and negative wishes.}

In often found examples of constraints with wishes (e.g., $[22,24]$ ), wishes have a positive flavor: if a wish is satisfied, it provides a bonus in favor of the item satisfying it against other items that have similar levels of satisfaction with respect to constraints, but do not satisfy this wish. Still, there may exist a negative counterpart to wishes. For instance, a request such as "a reasonably priced apartment, if possible not on the groundfloor" may not just mean that "not being on the groundfloor" provides a bonus, but rather that "being on the groundfloor" has a negative flavor. In fact, one may have both positive and negative wishes, as in "a reasonably priced apartment, if possible close to the train station, and if possible not on the groundfloor".

This calls for the use of a bipolar univariate scale where a neutral level separates the positive grades from the negative ones, and where the positive wishes and the negative wishes are handled separately. Then, one may for instance give priority to negative wishes, and use the negative wishes for breaking ties between items having similar levels of satisfaction with respect to constraints, and then use positive wishes for breaking further ties if any left. One may also think of combining evaluations pertaining to the positive wishes and the negative wishes, thus introducing compensation between them.

Another simpler option, already discussed in [2], would be to rank-order the items on the basis of the constraints and then for a given constraint satisfaction level to use the number of wishes satisfied (for instance the positive ones) for each item, providing the user with arguments pro and con respectively corresponding to the positive and to the negative wishes fulfilled by the item.

\section{Possibilistic logic modeling}

In this section, we reexamine the modeling of " and if possible" in a logical setting and enlarge the discus- sion to non conjunctive queries. We consider different forms of queries asking for items satisfying conditions $C_{1}, C_{2}, C_{3}$, with the information that $C_{1}$ is more important than $C_{2}$, which is itself more important than $C_{3}$. Conditions are supposed to be binary. They are not necessarily nested. They may be logically independent or not. For the sake of simplicity, we use here three conditions only, but what follows would straightforwardly extend to $n$ conditions. We denote by $\left[C_{i}\right],\left[C_{i} \wedge C_{j}\right]$, the set of items (if any) satisfying condition $C_{i}$, the set of items (if any) satisfying $C_{i}$ and $C_{j}$, and so on. We first consider conjunctive queries.

\subsection{Conjunctive queries}

Consider the query of the type " $C_{1}$ is required and if possible $C_{2}$ also and if possible $C_{3}$ too", with the following intended meaning ( $\gg$ reads "is preferred to") in terms of items:

$\left[C_{1} \wedge C_{2} \wedge C_{3}\right] \gg\left[C_{1} \wedge C_{2} \wedge \neg C_{3}\right] \gg\left[C_{1} \wedge \neg C_{2}\right] \gg\left[\neg C_{1}\right]$

i.e., one prefers to have the three conditions satisfied rather than the two first ones only, which is itself better than having just the first condition satisfied (which in turn is better than not having even the first condition satisfied).

This may be described in possibilistic logic [23] in different ways. First, it can be expressed as the conjunction of prioritized goals $\mathcal{C}=\left\{\left(C_{1}, \gamma_{1}\right),\left(C_{2}, \gamma_{2}\right),\left(C_{3}, \gamma_{3}\right)\right\}$ with $1=\gamma_{1}>\gamma_{2}>\gamma_{3}>0$. Indeed, this possibilistic logic base is associated with the possibility distribution

$$
\begin{aligned}
\pi_{\mathcal{C}}(\omega)= & 1 \text { if } \omega \in\left[C_{1} \wedge C_{2} \wedge C_{3}\right] \\
& 1-\gamma_{3} \text { if } \omega \in\left[C_{1} \wedge C_{2} \wedge \neg C_{3}\right] \\
& 1-\gamma_{2} \text { if } \omega \in\left[C_{1} \wedge \neg C_{2}\right] \\
& 0 \text { if } \omega \in\left[\neg C_{1}\right] .
\end{aligned}
$$

which fully agrees with the ordering (7).

Besides, in a logical encoding, a query such as "find the $x$ 's such that condition $Q$ is true", i.e., $\exists x Q(x)$ ? is usually processed by refutation. Using a small old trick due to Green [29], it amounts to adding the formula(s) corresponding to $\neg Q(x) \vee$ answer $(x)$, expressing that if item $x$ satisfies condition $Q$ it belongs to the answer, to the logical base describing the content of the database. It enables theorem-proving by resolution to be applied to question-answering. This idea extends to preference queries expressed in a possibilistic logic setting [11]. The expression of the query $\mathcal{Q}$ corresponding to the above set of prioritized goals is then of the form

$$
\begin{aligned}
\mathcal{Q}=\{ & \left(\neg C_{1}(x) \vee \neg C_{2}(x) \vee \neg C_{3}(x) \vee \text { answer }(x), 1\right), \\
& \left(\neg C_{1}(x) \vee \neg C_{2}(x) \vee \text { answer }(x), 1-\gamma_{3}\right), \\
& \left.\left(\neg C_{1}(x) \vee \text { answer }(x), 1-\gamma_{2}\right)\right\} .
\end{aligned}
$$


where $1>1-\gamma_{3}>1-\gamma_{2}$. Then, the levels associated with the possibilistic logic formulas expressing the preference query are directly associated with the possibility levels of the possibility distribution $\pi_{\mathcal{C}}$ providing its semantics.

\subsection{The two bipolar approaches in possibilistic logic}

Let us go back to the example considered in Section 3.2. We considered a request of the form " $\mathcal{A}$ and if

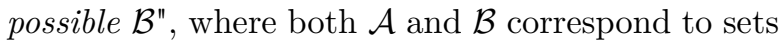
of prioritized goals, namely and respectively: and

$$
\mathcal{A}=\left\{\left(A_{2}, 1\right),\left(A_{1}, 1-\alpha\right)\right\} \text { with } 1>1-\alpha>0,
$$

$$
\mathcal{B}=\left\{\left(B_{2}, 1\right),\left(B_{1}, 1-\alpha^{\prime}\right)\right\} \text { with } 1>1-\alpha^{\prime}>0 .
$$

Remember we assumed $A_{2} \supseteq A_{1} \supseteq B_{1}$, $A_{2} \supseteq B_{2} \supseteq B_{1}$ and took $\alpha^{\prime}<\alpha<\lambda$, with $B_{2} \bigcap A_{2} \neq \emptyset$. Then, when both $\mathcal{A}$ and $\mathcal{B}$ are viewed as constraints, with priority to the ones associated with $\mathcal{A}$, the request " $\mathcal{A}$ and if possible $\mathcal{B}$ " translates into a unique set $\mathcal{G}$ of prioritized goals, where the goals in $B$ are discounted by $1-\lambda$ :

$$
\mathcal{G}=\left\{\left(A_{2}, 1\right),\left(A_{1}, 1-\alpha\right),\left(B_{2}, \min (1,1-\right.\right.
$$$$
\left.\lambda)),\left(B_{1}, \min \left(1-\alpha^{\prime}, 1-\lambda\right)\right)\right\}
$$

This possibilistic logic base is associated with the possibility distribution

$$
\begin{aligned}
\pi_{\mathcal{G}}(\omega)= & 1 \text { if } \omega \in\left[A_{1} \wedge B_{1}\right] \\
& \lambda \text { if } \omega \in\left[A_{1} \wedge \neg B_{1}\right] \\
& \alpha \text { if } \omega \in\left[A_{2} \wedge \neg A_{1} \wedge B_{2}\right] \\
& 0 \text { if } \omega \in\left[\neg A_{2}\right] .
\end{aligned}
$$

This corresponds exactly to the membership function of $F$ in Section 3.2.

Let us now consider the second view where only $\mathcal{A}$ is regarded as a set of prioritized constraints, while $\mathcal{B}$ is a set of prioritized wishes. Now we keep $\mathcal{A}$ and $\mathcal{B}$ separate. Each interpretation $\omega$ is the associated with a pair of values: the first (resp. the second) value is equal to $1-\gamma^{*}$ (resp. $1-\delta^{*}$ ) where $\gamma^{*}\left(\right.$ resp. $\left.\delta^{*}\right)$ is the priority of the formula violated by $\omega$ having the highest priority in $\mathcal{A}$ (resp. $\mathcal{B}$ ). We obtain, the following vector-valued possibility distribution:

$$
\begin{aligned}
\pi_{(\mathcal{A}, \mathcal{B})}(\omega)= & (1,1) \text { if } \omega \in\left[A_{1} \wedge B_{1}\right] \\
& \left(1, \alpha^{\prime}\right) \text { if } \omega \in\left[A_{1} \wedge \neg B_{1} \wedge B_{2}\right] \\
& (1,0) \text { if } \omega \in\left[A_{1} \wedge \neg B_{2}\right] \\
& \left(\alpha, \alpha^{\prime}\right) \text { if } \omega \in\left[A_{2} \wedge \neg A_{1} \wedge B_{2}\right] \\
& (\alpha, 0) \text { if } \omega \in\left[A_{2} \wedge \neg A_{1} \wedge \neg B_{2}\right] \\
& (0,0) \text { if } \omega \in\left[\neg A_{2}\right]
\end{aligned}
$$

Note the lexicographic ordering of the evaluation vectors. This corresponds to the 6 layers of interpretations found in 3.2 , and makes it clear that this second view is (trivially) more refined.

\subsection{Disjunctive queries}

We now consider disjunctive queries of the form "at least $C_{3}$ is required, or better $C_{2}$, or still better $C_{1}$ ", as discussed in [11] and in Section 2.2. It can be equivalently stated starting with what is preferred: " $C_{1}$ is required with priority, or failing this $C_{2}$, or still failing this $C_{3}$ ". It has the following intended meaning in terms of interpretations:

$\left[C_{1}\right] \gg\left[\neg C_{1} \wedge C_{2}\right] \gg\left[\neg C_{1} \wedge \neg C_{2} \wedge C_{3}\right] \gg\left[\neg C_{1} \wedge \neg C_{2} \wedge \neg C_{3}\right]$.

As can be checked, it corresponds to the following possibilistic logic base representing a conjunction of prioritized goals:

$$
\mathcal{D}=\left\{\left(C_{1} \vee C_{2} \vee C_{3}, 1\right),\left(C_{1} \vee C_{2}, \gamma_{2}\right),\left(C_{1}, \gamma_{3}\right)\right\} .
$$

(with $\gamma_{1}=1>\gamma_{2}>\gamma_{3}$ ) whose associated possibility distribution is

$$
\begin{aligned}
\pi_{\mathcal{D}}(\omega)= & 1 \text { if } \omega \in\left[C_{1}\right] \\
& 1-\gamma_{3} \text { if } \omega \in\left[\neg C_{1} \wedge C_{2}\right] \\
& 1-\gamma_{2} \text { if } \omega \in\left[\neg C_{1} \wedge \neg C_{2} \wedge C_{3}\right] \\
& 0 \text { if } \omega \in\left[\neg C_{1} \wedge \neg C_{2} \wedge \neg C_{3}\right],
\end{aligned}
$$

which is clearly in agreement with the ordering (8). It can be also equivalently expressed in a questionanswering perspective by the possibilistic logic base:

$$
\begin{aligned}
\mathcal{Q}^{\prime}= & \left\{\left(\neg C_{1}(x) \vee \text { answer }(x), 1\right),\right. \\
& \left(\neg C_{2}(x) \vee \text { answer }(x), 1-\gamma_{3}\right), \\
& \left.\left(\neg C_{3}(x) \vee \text { answer }(x), 1-\gamma_{2}\right)\right\} .
\end{aligned}
$$

which states that if an item $x$ satisfies $C_{1}$, then it belongs to the answer to degree 1 , and if it satisfies $C_{2}$ (resp. $C_{3}$ ), then it belongs to the answer to a degree at least equal to $1-\gamma_{3}\left(\operatorname{resp} 1-\gamma_{2}\right)$.

Let us also explain the relation between the possibilistic representation and qualitative choice logic (QCL) [13]. Indeed QCL introduces a new connective denoted $\times$, where $C_{1} \times C_{2}$ means "if possible $C_{1}$, but if $C_{1}$ is impossible then (at least) $C_{2}$ ". This corresponds to a disjunctive preference of the above type. Then, the query " $C_{1}$, or at least $C_{2}$, or at least $C_{3}$ ", which, as already explained, corresponds to stating that $C_{1}$ is fully satisfactory, $C_{2}$ instead is less satisfactory, and $C_{3}$ instead is still less satisfactory, can be directly represented in a non classical possibilistic logic (see [3]) based on guaranteed possibility measures, rather than on necessity measures. Using the notation $\left[C_{i}, \alpha_{i}\right]$ for $\Delta\left(C_{i}\right) \geq \alpha_{i}$, the corresponding weighted base simply writes $\mathcal{D}_{\Delta}=\left\{\left[C_{1}, 1\right],\left[C_{2}, 1-\gamma_{3}\right],\left[C_{3}, 1-\gamma_{2}\right]\right\}$, which clearly echoes $\mathcal{Q}^{\prime}$, and encodes the same possibility distributions on models as $\mathcal{D}$.

Note that in $\mathcal{Q}^{\prime}$, as in $\mathcal{Q}$, the weights of the possibilistic logic formulas express a priority among the answers $x$ that may be obtained. They may be also viewed as representing the levels of satisfaction of the answers obtained. Besides, an extension of QCL 
allows for the use of prioritized conjunctions [5], in a way agreeing with possibilistic logic.

\subsection{Relation between conjunctive and disjunctive queries}

The linguistic expression of conjunctive queries may suggest that $C_{1}, C_{2}, C_{3}$ are logically independent conditions that one would like to cumulate, as in the query "I am looking for a reasonably priced hotel, if possible downtown, and if possible not far from the station", while in disjunctive queries one may think of $C_{3}$ as a relaxation of $C_{2}$, itself a relaxation of $C_{1}$. In fact there is no implicit limitation on the type of conditions involved in conjunctive or disjunctive queries. For instance, a conjunctive query such as "I am looking for a hotel less than $2 \mathrm{~km}$ from the beach, if possible less than $1 \mathrm{~km}$ from the beach, and if possible on the beach", corresponds to the idea of approximating a fuzzy requirement, such as "close to the beach" by three of its level cuts, which are then relaxation or strengthening of one another.

As noticed in [11] and in section 2.3, there is a perfect duality between conjunctive and disjunctive queries. Indeed the disjunctive query " $C_{3}$ is required, or better $C_{2}$, or still better $C_{1}$ " can be also equivalently expressed under the conjunctive form " $C_{1}$ or $C_{2}$ or $C_{3}$ is required and if possible $C_{1}$ or $C_{2}$, and if possible $C_{1}$ ". This can be checked by noticing that changing $C_{1}$ into $C_{1} \vee C_{2} \vee C_{3}, C_{2}$ into $C_{1} \vee C_{2}$, and $C_{3}$ in $C_{1},(7)$ is changed into (8). Conversely, the conjunctive query " $C_{1}$ is required and if possible $C_{2}$ and if possible $C_{3}$ " can be equivalently stated as the disjunctive query " $C_{1}$ is required, or better $C_{1}$ and $C_{2}$, or still better $C_{1}$ and $C_{2}$ and $C_{3}$ ". It can be checked that changing $C_{1}$ into $C_{1} \wedge C_{2} \wedge C_{3}, C_{2}$ into $C_{1} \wedge C_{2}$ and $C_{3}$ in $C_{1},(8)$ is changed into (7). The duality between the two types of queries, laid bare in section 2.3, can be checked as well on their respective possibilistic logic representations.

\section{Concluding remarks}

This paper presents a detailed study of an issue closely related to the ideas of gradualness and bipolarity [27], namely the representation of requirements of the form " $A$ and if possible $B$ " or of a related form. We have emphasized the existence of two different views, according to whether $B$ plays, or does not play, the role of a constraint of the same kind as $A$. We have discussed the logical expression of such requirements, and provided a unified presentation of a fuzzy logic-based approach together with other approaches to preference queries.

\section{References}

[1] K. Abbaci, F. Lemos, A. Hadjali, D. Grigori, L. Liétard, D. Rocacher, M. Bouzeghoub. A bipolar approach to the handling of user preferences in business processes retrieval. In: Advances on Computational Intelligence, (S. Greco, et al., eds.), Proc. 14th Int. Conf. on Information Processing and Management of Uncertainty in Knowledge-based Systems (IPMU'12), Catania, July 9-13, 2012, Springer, CCIS series, 400-409.

[2] L. Amgoud, H. Prade, M. Serrut. Flexible querying with argued answers. Proc. 14th IEEE Inter. Conf. on Fuzzy Systems (FUZZ'05), 573-578, 2005.

[3] S. Benferhat, G. Brewka, D. Le Berre. On the relation between qualitative choice logic and possibilistic logic. Proc. 10th Int. Conf. on Information Processing and Management of Uncertainty in Knowledge-Based Systems (IPMU'04), Perugia, July 4-9, 951-957, 2004.

[4] S. Benferhat, D. Dubois, S. Kaci, H. Prade. Modeling positive and negative information in possibility theory. Int. J. of Intelligent Systems, 23 (10),1094-1118, 2008.

[5] S. Benferhat, K. Sedki. Two alternatives for handming preferences in qualitative choice logic. Fuzzy Sets and Systems, 159,1889-1912, 2008.

[6] P. Bosc, O. Pivert. Some approaches for relational databases flexible querying. J. Intell. Inf. Syst., 1 (3/4), 323-354, 1992.

[7] P. Bosc, O. Pivert. SQLf: a relational database language for fuzzy querying. IEEE Trans. on Fuzzy Systems, 3, 1995, 1-17.

[8] P. Bosc, O. Pivert. On diverse approaches to bipolar division operators. Int. J. Intell. Syst., 26 (10), 911-929, 2011.

[9] P. Bosc, O. Pivert. On four noncommutative fuzzy connectives and their axiomatization. Fuzzy sets and Systems, 202, 42-60, 2012.

[10] P. Bosc, O. Pivert, A. Mokhtari, L. Liétard. Extending relational algebra to handle bipolarity. Proc. 2010 ACM Symp. on Applied Computing (SAC), Sierre, Switz., (S. Y. Shin, S. Ossowski, M. Schumacher, M. J. Palakal, C.C. Hung, eds.), March 22-26,1718-1722, 2010.

[11] P. Bosc, O. Pivert, H. Prade. A possibilistic logic view of preference queries to an uncertain database. Proc. 19th IEEE Inter. Conf. on Fuzzy Systems (FUZZ-IEEE'10), Barcelona, July 18-23,1-6, 2010.

[12] P. Bosc, O. Pivert, O. Soufflet. On three classes of division queries involving ordinal preferences. J. Intell. Inf. Syst. 37(3): 315-331 (2011)

[13] G. Brewka, S. Benferhat, D. Le Berre. Qualitative choice logic. Artificial Intelligence, 157 (1-2), 203-237, 2004.

[14] J. Chomicki. Preference formulas in relational queries. ACM Transactions on Database Systems, 28,1-40, 2003.

[15] M. de Calmès, D. Dubois, E. Hüllermeier, H. Prade, F. Sèdes. Flexibility and fuzzy casebased evaluation in querying: An illustration in an experimental setting. Inter. J. of Un- 
certainty, Fuzziness and Knowledge-Based Systems, 11 (1), 43-66, 2003.

[16] R. Confalonieri, H. Prade. Encoding preference queries to an uncertain database in possibilistic answer set programming. Proc. 14th Int. Conf. on Information Processing and management of Uncertainty in Knowledge-based Systems (IPMU'12), Catania, July 9-13, 2012.

[17] C. Domshlak, E. Hüllermeier, S. Kaci, H. Prade. Preferences in AI: An overview. Artificial Intelligence, 175 (7-8), 1037-1052, 2011.

[18] D. Dubois, H. Fargier, H. Prade. Possibility theory in constraint satisfaction problems: Handling priority, preference and uncertainty. Applied Intelligence, 6, 1996, 287-309.

[19] D. Dubois, H. Prade. Weighted minimum and maximum operations, Information Sciences, 39, 205-210, 1986.

[20] D. Dubois, H. Prade. Default reasoning and possibility theory. Artificial Intelligence, 35 (2), 243-257, 1988.

[21] D. Dubois, H. Prade. Using fuzzy sets in flexible querying: Why and how? In: Flexible Query Answering Systems (T. Andreasen, H. Christiansen, H. L. Larsen, eds.), Kluwer Acad. Publ., 45-60, 1997.

[22] D. Dubois, H. Prade. Bipolarity in flexible querying. Proc. 5th Inter. Conf. on Flexible Query Answering Systems (FQAS'02), (T. Andreasen, A. Motro, H. Christiansen, H. L. Larsen, eds.), Copenhagen, Oct. 27-29, Springer, LNCS 2522, 174-182, 2002.

[23] D. Dubois, H. Prade. Possibilistic logic: a retrospective and prospective view. Fuzzy Sets and Systems, 144 (1), 3-23, 2004.

[24] D. Dubois, H. Prade. Handling bipolar queries in fuzzy information processing. In: Handbook of Research on Fuzzy Information Processing in Databases, (J. Galindo, ed.), IGI Global, 97114, 2008.

[25] D. Dubois, H. Prade. An introduction to bipolar representations of information and preference. Int. J. of Intelligent Systems, 23 (8), 866$877,2008$.

[26] D. Dubois, H. Prade. An overview of the asymmetric bipolar representation of positive and negative information in possibility theory. Fuzzy Sets and Systems, 160 (10), 1355-1366, 2009.

[27] D. Dubois, H. Prade. Gradualness, uncertainty and bipolarity: Making sense of fuzzy sets. Fuzzy Sets and Systems, 192, 3-24, 2012.

[28] D. Dubois, H. Prade. Modeling "and if possible" and "or at least": Different forms of bipolarity in flexible querying. In: Flexible Approaches to Data Management, a volume dedicated to Patrick Bosc, (O. Pivert, S. Zadrozny, eds.), Springer, to appear

[29] C. Green. Theorem-proving by resolution as a basis for question-anwering systems. In: Ma- chine Intelligence, Vol. 4, (D. Michie and B. Meltzer, eds.), Edinburgh University Press, 1969, 183-205.

[30] A. Hadjali, S. Kaci, H. Prade. Database preference queries - A possibilistic logic approach with symbolic priorities. (Preliminary version in Proc. 5th Int. Symp. on Foundations of Information and Knowledge Systems (FolKS 2008), Pisa, Feb. 11-14, (S. Hartmann, G. Kern-Isberner, eds.), Springer, LNCS 4932, 291-310, 2008). Annals of Mathematics and Artificial Intelligence, 63 (3-4), 357-383, 2011.

[31] W. Kiessling. Foundations of preferences in database systems. Proc. 28th Inter. Conf. on Very Large Data Bases (VLDB'02), 311-322, 2002.

[32] M. Lacroix, P. Lavency. Preferences: putting more knowledge into queries. Proc. 13th Conf. on Very Large Data Bases (VLDB'87), 217 225, 1987.

[33] L. Liétard, N. Tamani, D. Rocacher: Fuzzy bipolar conditions of type "or else". Proc. 20th IEEE Inter. Conf. on Fuzzy Systems (FUZZIEEE'11), Taipei, June, 27-30, 2546-2551, 2011.

[34] T. Lukasiewicz and J. Schellhase. Variablestrength conditional preferences for ranking objects in ontologies. J. Web Semantics, 5 (3), 180-194, 2007.

[35] A. Motro. A user interface to relational databases that permits vague queries. ACM Transactions on Information Systems, 6, 187214, 1988.

[36] O. Pivert, P. Bosc. Fuzzy Preference Queries to Relational Databases. Imperial College Press, 2012.

[37] V. Tahani. A conceptual framework for fuzzy query processing - A step toward very intelligent database systems. Information Processing and Management,12, 289-303, 1977.

[38] R. R. Yager. Non-monotonic set theoretic operations. Fuzzy Sets and Systems, 42, 173-190, 1991.

[39] L. A. Zadeh. Fuzzy sets, Information Control, 8, 338-353, 1965.

[40] S. Zadrozny, J. Kacprzyk. Bipolar queries using various interpretations of logical connectives. In: Foundations of Fuzzy Logic and Soft Computing, Proc. 12th International Fuzzy Systems Association World Congress (IFSA'07), Cancun, June 18-21, (P. Melin, O. Castillo, L. T. Aguilar, J. Kacprzyk, W. Pedrycz, eds.), Springer Verlag, LNCS 4529, 181-190, 2007.

[41] S. Zadrozny, G. De Tré, R. De Caluwe, J. Kacprzyk. An overview of fuzzy approaches to flexible database querying. In: Database Technologies: Concepts, Methodologies, Tools, and Applications (4 Volumes), (J. Erickson, ed.), IGI Global, 135-156, 2009. 